\title{
Multipole moments of heavy vector and axial-vector mesons in QCD
}

\author{
T. M. Aliev $\odot,{ }^{*}$ S. Bilmis $\odot,^{\dagger}$ and M. Savci ${ }^{\ddagger}$ \\ Department of Physics, Middle East Technical University, Ankara 06800, Turkey
}

(Received 16 December 2019; accepted 24 February 2020; published 9 March 2020)

\begin{abstract}
The magnetic and quadrupole moments of the vector and axial-vector mesons containing a heavy quark are estimated within the light-cone sum rules method. Our predictions on magnetic moments for the vector mesons are compared with the results obtained by other approaches.
\end{abstract}

DOI: 10.1103/PhysRevD.101.054009

\section{INTRODUCTION}

A study of the electromagnetic properties of hadrons plays a crucial role in understanding their inner structure. The magnetic moments are one of the fundamental characteristics of hadrons. The magnetic moments of hadrons are related to their magnetic form factors; more precisely, the magnetic moment is equal to the magnetic form factor at zero momentum square. The magnetic moments of mesons have not received much interest compared to baryons except the $\rho$ meson, which has been intensively studied in the literature within different approaches [1-11]. The magnetic moments of $K^{*}$ mesons have also been investigated in several works $[1-3,5,6,9,10]$. On the other hand, the magnetic moments of heavy mesons have been calculated only in a few works $[10,12,13]$. In the face of this situation, it is timely to study the magnetic moments of heavy vector and axial-vector mesons. It is challenging to measure the magnetic moments of vector mesons directly, since their lifetimes are very short. Even though indirect measurement is possible [14], it has a large uncertainty. It should be noted that with the help of the magnetic dipole transitions $M 1$, for which there exist many experimental data, it is possible to determine the transition magnetic moments of heavy mesons. There are lots of theoretical works, such as the quark model $[15,16]$, nonrelativistic QCD [17], the quark potential model [18-21], various relativistic models, the bag model [22-24], the light front model [25-27], the Bethe-Salpeter equation [28,29], QCD sum rules [30-38], lattice QCD [39,40], the chiral model [41-45], the Nambu-Jona-Lasinio model, the dispersion approach, etc., devoted to this subject.

\footnotetext{
*taliev@metu.edu.tr

†sbilmis@metu.edu.tr

*savci@metu.edu.tr
}

Published by the American Physical Society under the terms of the Creative Commons Attribution 4.0 International license. Further distribution of this work must maintain attribution to the author(s) and the published article's title, journal citation, and DOI. Funded by SCOAP.
In the present work, we calculate the magnetic moments of heavy vector and axial-vector mesons within lightcone QCD sum rules (LCSR) [46]. The calculation of the multipole moments for axial-vector mesons is performed for the first time.

The paper is organized as follows. In Sec. II, we construct the LCSR for multipole moments of heavy vector and axial-vector mesons. The following section is devoted to the numerical analysis of the sum rules for the multipole moments of heavy vector and axial-vector mesons. In this section, the obtained results are also compared with predictions of other approaches in the literature. The last section contains a summary and discussions.

\section{LIGHT-CONE SUM RULES FOR MULTIPOLE MOMENTS}

The LCSR for multipole moments of vector (axialvector) heavy mesons can be obtained by considering the following correlation function:

$$
\Pi^{\mu \alpha \nu}=i^{2} \int d^{4} x \int d^{4} y e^{i p x+i q y}\left\langle 0\left|T\left\{J^{\mu}(x) j_{e l}^{\alpha}(y) J^{\nu^{\dagger}}(0)\right\}\right| 0\right\rangle,
$$

where $J^{\mu}(x)=\bar{q}^{a}(x) \gamma_{\mu} Q^{a}(x)$ is the interpolating current with the quantum numbers of a heavy vector meson and $a$ is the color index. The interpolating current for axial-vector mesons can be obtained from $J^{\mu}(x)$ with the simple replacement $\gamma^{\mu} \rightarrow \gamma^{\mu} \gamma^{5}$. The current $j_{e l}^{\alpha}(y)=e_{q} \bar{q} \gamma^{\alpha} q+e_{Q} \bar{Q} \gamma^{\alpha} Q$ is the electromagnetic current, and $e_{q}$ and $e_{Q}$ are the electric charges of the light and heavy mesons, respectively.

The general strategy of QCD sum rules is that the correlation function has to be calculated in different kinematical domains. In one domain, it is saturated by the corresponding heavy vector (axial-vector) mesons, i.e., $p^{2} \simeq m_{V_{Q}}^{2}\left(m_{A_{Q}}^{2}\right)$ (hadronic part). In the other domain, where $p^{2} \ll 0,(p+q)^{2} \ll 0$, the calculation is performed by using the operator product expansion (OPE) in terms of the 
photon distribution amplitudes (DAs) with an increasing twist. Matching the results of these representations, one can get the desired sum rules for the relevant physical quantities.
The hadronic part of the correlation function can be obtained by inserting a complete set of states carrying the same quantum numbers of the interpolating currents. Isolating the ground state vector mesons, we have

$$
\Pi_{\mu \nu \alpha}(p, q)=\frac{\left\langle 0\left|J_{\mu}\right| i(p)\right\rangle\left\langle i(p)\left|j_{\alpha}^{e l}(y)\right| f\left(p^{\prime}\right)\right\rangle\left\langle f\left(p^{\prime}\right)\left|J_{\nu}^{+}\right| 0\right\rangle+\cdots}{\left(p^{2}-m_{i}^{2}\right)\left(p^{\prime 2}-m_{i}^{2}\right)} .
$$

The matrix element $\left\langle 0\left|J_{\mu}\right| i(p)\right\rangle$ in Eq. (2) is defined as

$$
\left\langle 0\left|J_{\mu}\right| i(p)\right\rangle=f_{i} m_{i} \epsilon_{\mu},
$$

where $f_{i}$ is the leptonic decay constant of the corresponding heavy vector mesons and $\epsilon_{\mu}$ is its polarization vector. Using the parity and time-reversal invariance of the electromagnetic interaction, the matrix element of the electromagnetic current between two vector (axialvector) mesons is described in terms of three form factors as [47]

$$
\left\langle f\left(p^{\prime}, \epsilon^{\prime}\right)\left|j_{\alpha}^{e l}\right| i\left(p, \epsilon^{r}\right)\right\rangle=-\left(\epsilon^{r}\right)^{\rho}\left(\epsilon^{r^{\prime}}\right)^{\beta}\left\{G_{1}\left(Q^{2}\right) g_{\rho \beta}\left(p^{\prime}+p\right)_{\alpha}+G_{2}\left(Q^{2}\right)\left(q_{\rho} g_{\alpha \beta}-q_{\beta} g_{\alpha \rho}\right)+\frac{G_{3}\left(Q^{2}\right)}{2 m_{i}^{2}} q_{\rho} q_{\beta}\left(p+p^{\prime}\right)_{\alpha}\right\},
$$

where $G_{i}$ are the form factors and $Q^{2}=-q^{2}$. The form factors $G_{i}\left(Q^{2}\right)$ are related to the charge, magnetic, and quadrupole multipole form factors in the following way [47]:

$$
\begin{aligned}
F_{c}\left(Q^{2}\right) & =G\left(Q^{2}\right)+\frac{2}{3} \eta F_{D}\left(Q^{2}\right), \\
F_{M}\left(Q^{2}\right) & =G_{2}\left(Q^{2}\right), \\
F_{D}\left(Q^{2}\right) & =G_{1}\left(Q^{2}\right)-G_{2}\left(Q^{2}\right)+(1+\eta) G_{3}\left(Q^{2}\right),
\end{aligned}
$$

where $\eta=Q^{2} / 4 m_{i}^{2}$ and $F_{C}\left(Q^{2}\right), F_{M}\left(Q^{2}\right)$, and $F_{D}\left(Q^{2}\right)$ are the charge, magnetic, and quadrupole form factors, respectively. The value of $F_{c}\left(Q^{2}\right), F_{M}\left(Q^{2}\right)$, and $F_{D}\left(Q^{2}\right)$ at the $Q^{2}=-q^{2}=0$ point gives the charge, magnetic moment $\mu$, and quadrupole moment $D$ of the vector (axial-vector) mesons.

Substituting Eqs. (3)-(5) into Eq. (2) and performing a summation over the spins of vector mesons for the hadronic part of the correlation function, we get

$$
\begin{aligned}
\Pi_{\mu \alpha \nu} \epsilon_{\gamma}^{\alpha}= & f_{i}^{2} m_{i}^{2} \frac{\epsilon_{\gamma}^{\alpha}}{\left(m_{i}^{2}-p^{2}\right)\left(m_{i}^{2}-(p+q)^{2}\right)} \\
& \times\left\{2 F_{c}(0) p_{\alpha}\left(g_{\mu \nu}-\frac{p_{\mu} p_{\nu}}{m_{i}^{2}}-\frac{p_{\mu} q_{\nu}}{m_{i}^{2}}\right)\right. \\
& +F_{M}(0)\left[q_{\mu} g_{\nu \alpha}-q_{\nu} g_{\mu \alpha}-\frac{p_{\alpha}}{m_{i}^{2}}\left(p_{\mu} q_{\nu}-p_{\nu} q_{\mu}\right)\right] \\
& \left.-\left[F_{c}(0)+F_{D}(0)\right] \frac{p_{\alpha}}{m_{i}^{2}} q_{\nu} q_{\mu}\right\}
\end{aligned}
$$

where $\epsilon_{\gamma}$ is the photon polarization vector. To derive this expression, the transversality condition $q \epsilon=0$ is used.

Now let us turn our attention to the calculation of Eq. (1) from the OPE side. By introducing the electromagnetic background field of a plane wave,

$$
F_{\mu \nu}=i\left(e_{\nu}^{\gamma} q_{\mu}-e_{\mu}^{\gamma} q_{\nu}\right) e^{i q x},
$$

the correlation function can be written in the following way:

$$
\Pi_{\mu \alpha \nu} \epsilon_{\gamma}^{\alpha}=i \int d^{4} x e^{i p x}\left\langle 0\left|T\left\{J_{\mu}(x) J_{\nu}^{+}(0)\right\}\right| 0\right\rangle_{F} .
$$

In this expression, the subscript $F$ means that the vacuum expectation value is evaluated in the presence of the background field $F_{\mu \nu}$. The correlation function given in Eq. (1) can be obtained from Eq. (8) by expanding it in linear powers of $F_{\mu \nu}$. More details about the background field method are given in two excellent reviews $[48,49]$.

Using the explicit expressions of the interpolating currents and applying the Wick theorem for the correlation function, we obtain

$$
\Pi_{\mu \alpha \nu} \epsilon_{\gamma}^{\alpha}=i \int d^{4} x e^{i p x}\left\langle 0\left|S_{Q}(x) \gamma_{\mu} S(-x) \gamma_{\nu}\right| 0\right\rangle_{F} .
$$

From this expression, it follows that, to calculate the correlation function in the deep Euclidean domain, it is necessary to know the explicit expressions of the light and heavy quark propagators in the presence of the background gluonic and electromagnetic fields. The expressions of these propagators are obtained in Refs. [50,51]: 


$$
\begin{aligned}
& S(x)=\frac{i \not x}{2 \pi^{2} x^{4}}-\frac{m_{q}}{4 \pi^{2} x^{2}}-\frac{i g_{s}}{16 \pi^{2} x^{2}} \int_{0}^{1} d u\left\{\bar{u} \not \sigma_{\alpha \beta}+u \sigma_{\alpha \beta} \not x\right\} G^{\alpha \beta}(u x) \frac{-i e_{q}}{16 \pi^{2} x^{2}} \int_{0}^{1} d u\left\{\bar{u} \not \sigma_{\alpha \beta}+u \sigma_{\alpha \beta} \not\right\} F^{\alpha \beta}(u x) \\
& -i g_{s} \int_{0}^{1} d u\left\{-\frac{i m_{q}}{32 \pi^{2}} G_{\mu \nu}(u x) \sigma^{\mu \nu} \ln \left(-\frac{x^{2} \Lambda^{2}}{u}+2 \gamma_{E}\right)\right\}-i e_{q} \int_{0}^{1} d u\left\{-\frac{i m_{q}}{32 \pi^{2}} F_{\mu \nu}(u x) \sigma^{\mu \nu} \ln \left(-\frac{x^{2} \Lambda^{2}}{u}+2 \gamma_{E}\right)\right\}, \\
& S_{Q}(x)=\int \frac{d^{4} k}{2 \pi^{4} i} e^{-i k x} \frac{\not k+m_{Q}}{m_{Q}^{2}-k^{2}}-i g_{s} \int \frac{d^{4} k}{2 \pi^{4} i} e^{-i k x} \int_{0}^{1} d u\left\{\frac{\not k+m_{Q}}{2\left(m_{Q}^{2}-k^{2}\right)^{2}} G^{\alpha \beta} \sigma_{\alpha \beta}+\frac{u x_{\alpha}}{m_{Q}^{2}-k^{2}} G^{\alpha \beta}(u x) \gamma_{\beta}\right\} \\
& -i e_{Q} \int \frac{d^{4} k}{2 \pi^{4} i} e^{-i k x} \int_{0}^{1} d u\left\{\frac{\not k+m_{Q}}{2\left(m_{Q}^{2}-k^{2}\right)} F^{\alpha \beta}(u x) \sigma_{\alpha \beta}+\frac{u x_{\alpha}}{m_{Q}^{2}-k^{2}} F^{\alpha \beta} \gamma_{\beta}\right\} \text {, }
\end{aligned}
$$

where $\bar{u}=1-u, \Lambda=(0.5 \pm 0.1) \mathrm{GeV}$ [52] is the scale parameter separating the perturbative and nonperturbative domains, and $\gamma_{E}=0.577$ is the Euler constant. Note that the four quark particle $\bar{q} q \bar{q} q$ and $\bar{q} G^{2} q$ operator contributions are small and are not presented in Eq. (10) [50,53].

In light-cone sum rules, the nonperturbative contribution appears when a photon is emitted at long distances. To obtain these contributions, it is necessary to expand the quark propagator near the light cone $x^{2}=0$. In this case, the following matrix elements of nonlocal operators in the presence of the external background field need to be evaluated:

$$
\begin{aligned}
& \langle 0|\bar{q}(x) \Gamma q(0)| 0\rangle_{F}, \\
& \left\langle 0\left|\bar{q}(x) \Gamma G_{\alpha \beta} q(0)\right| 0\right\rangle_{F}, \\
& \left\langle 0\left|\bar{q}(x) \Gamma F_{\mu \nu} q(0)\right| 0\right\rangle_{F},
\end{aligned}
$$

where $\Gamma$ is arbitrary Dirac matrices. These matrix elements are described by photon distribution amplitudes, which were determined in Ref. [49] and are presented in Appendix A for completeness.

From Eq. (6), it follows that we have numerous structures which can be used to calculate the magnetic and quadrupole moments of heavy vector (axial-vector) mesons. We adopt the structures $(p \epsilon) p_{\mu} p_{\nu}$ and $(p \epsilon) p_{\nu} q_{\mu}$ to determine $F_{c}(0), F_{M}(0)$, and $F_{c}(0)+F_{D}(0)$. The choice of these structures is dictated by the fact that they contain the maximal number of momenta, which exhibits good convergence, in general. The theoretical part of the correlation function can be obtained from Eq. (9) by substituting the explicit expressions of the heavy and light quark propagators and the photon DAs. Performing an integration over $x$, the expression of the correlation function in the momentum representation can be obtained. Matching these two expressions of the correlation function via the dispersion relation and performing doubly Borel transformations on $-p^{2}$ and $-(p+q)^{2}$ in order to suppress the contributions of higher states and continuum, we get the desired sum rules for the multipole form factors. Note that the higher state contributions are taken into account by using the quark-hadron duality ansatz.
In result, we get the following sum rules for the charge $F_{c}(0)$, magnetic moment $F_{M}(0)$, and the sum of charge and quadrupole moment form factors at the $Q^{2}=0$ point:

$$
\begin{aligned}
F_{c}(0) & =-\frac{1}{2 f_{i}^{2}} e^{m_{i}^{2} / M^{2}} \Pi_{1}^{( \pm)}, \\
F_{M}(0) & =-\frac{1}{f_{i}^{2}} e^{m_{i}^{2} / M^{2}} \Pi_{2}^{( \pm)}, \\
F_{c}(0)+F_{D}(0) & =-\frac{1}{f_{i}^{2}} e^{m_{i}^{2} / M^{2}} \Pi_{3}^{( \pm)} .
\end{aligned}
$$

Explicit expressions of $\Pi_{1}^{( \pm)}, \Pi_{2}^{( \pm)}$, and $\Pi_{3}^{( \pm)}$are presented in Appendix B. The upper (lower) sign corresponds to vector (axial-vector) mesons. Moreover, we denote $D_{1}$ and $D_{s 1}$ axial-vector mesons with mass 2420 and $2460 \mathrm{MeV}$, respectively.

\section{NUMERICAL ANALYSIS}

This section is devoted to the numerical analysis of the sum rules for the multipole moments of the heavy vector (axial-vector) mesons. The values of the input parameters entering the sum rules are presented in Table I. In this study, we use the $\overline{\mathrm{MS}}$ mass, $m_{c}\left(m_{c}\right)=(1.275 \pm 0.035 \mathrm{GeV})$, $m_{b}\left(m_{b}\right)=(4.18 \pm 0.03 \mathrm{GeV})$, and take into account the scale dependence of the $\overline{\mathrm{MS}}$ masses coming from the renormalization group equation:

$$
\begin{aligned}
& m_{b}(\mu)=m_{b}\left(m_{b}\right)\left(\frac{\alpha_{s}(\mu)}{\alpha_{s}\left(m_{b}\right)}\right)^{12 / 23}, \\
& m_{c}(\mu)=m_{c}\left(m_{c}\right)\left(\frac{\alpha_{s}(\mu)}{\alpha_{s}\left(m_{c}\right)}\right)^{12 / 25} .
\end{aligned}
$$

Besides the input parameters that are presented in Table I, sum rules contain two more extra parameters, namely, the continuum threshold $s_{0}$ and the Borel mass parameter $M^{2}$. The domain of $M^{2}$ is determined by demanding the standard criteria; namely, both power corrections and continuum contributions should be sufficiently suppressed. The continuum threshold is determined from the condition 
TABLE I. The values of the input parameters.

\begin{tabular}{ll}
\hline \hline$\langle\bar{q} q\rangle(1 \mathrm{GeV})$ & $\left(-0.246_{-0.019}^{+0.028}\right)^{3} \mathrm{GeV}^{3}[54]$ \\
$\langle\bar{s} s\rangle(1 \mathrm{GeV})$ & $0.8 \times\langle\bar{q} q\rangle[54]$ \\
$m_{0}^{2}$ & $(0.8 \pm 0.2) \mathrm{GeV}^{2}[55]$ \\
$m_{s}(2 \mathrm{GeV})$ & $\left(96_{-4}^{+8} \times 10^{-3}\right) \mathrm{GeV}[56]$ \\
$f_{\mathcal{D}^{*}}$ & $(0.263 \pm 0.021) \mathrm{GeV}[57]$ \\
$f_{\mathcal{D}_{s}^{*}}$ & $(0.308 \pm 0.021) \mathrm{GeV}[57]$ \\
$f_{B^{*}}$ & $\left(0.196_{-0.027}^{+0.028}\right) \mathrm{GeV}[57]$ \\
$f_{B_{s}^{*}}$ & $(0.255 \pm 0.019) \mathrm{GeV}[57]$ \\
$f_{\mathcal{D}_{1}}$ & $(0.332 \pm 0.018) \mathrm{GeV}[57]$ \\
$f_{\mathcal{D}_{s 1}}$ & $(0.245 \pm 0.017) \mathrm{GeV}[57]$ \\
$f_{B_{1}}$ & $(0.335 \pm 0.018) \mathrm{GeV}^{*}[57]$ \\
$f_{B_{s 1}}$ & $(0.348 \pm 0.018) \mathrm{GeV}[57]$ \\
$\chi(1 \mathrm{GeV})$ & $-(2.85 \pm 0.5) \mathrm{GeV}^{-2}[48]$ \\
$f_{3 \gamma}$ & $-0.0039 \mathrm{GeV}^{-2}[49]$ \\
\hline \hline
\end{tabular}

TABLE II. Working region of $M^{2}$ and $s_{0}$ parameters are shown.

\begin{tabular}{lcc}
\hline \hline Mesons & $M^{2}\left(\mathrm{GeV}^{2}\right)$ & $s_{0}\left(\mathrm{GeV}^{2}\right)$ \\
\hline$B^{*}$ & $(11 \pm 3)$ & $(35 \pm 1)$ \\
$B_{s}^{*}$ & $(12 \pm 3)$ & $(37 \pm 1)$ \\
$B_{1}$ & $(13 \pm 2)$ & $(42 \pm 1)$ \\
$B_{s 1}$ & $(14 \pm 3)$ & $(43 \pm 1)$ \\
$D^{*}$ & $(4.5 \pm 1.5)$ & $(6.5 \pm 0.5)$ \\
$D_{s}^{*}$ & $(4.5 \pm 1.5)$ & $(7.5 \pm 0.5)$ \\
$D_{1}$ & $(5 \pm 2)$ & $(8.5 \pm 0.5)$ \\
$D_{s 1}$ & $(5 \pm 2)$ & $(9.5 \pm 0.5)$ \\
\hline \hline
\end{tabular}

that the sum rules should reproduce the mass of the ground state mass with $10 \%$ accuracy. These conditions are fulfilled in the regions of $M^{2}$ and $s_{0}$ presented in Table II. Having specified all input parameters, we are ready to calculate the numerical values of the magnetic and quadrupole moments, i.e., corresponding form factors at the $q^{2}=$ 0 point of all considered vector and axial-vector mesons.

In Figs. 1 and 2, we presented the dependency of $F_{M}^{D^{*+}}$ and $F_{M}^{B^{*+}}$ on $M^{2}$ at two fixed values of the continuum threshold, respectively, for illustration. From these figures, we observe good stability of $F_{M}^{D^{*+}}$ and $F_{M}^{B^{*+}}$ to the variation of $M^{2}$. In Fig. 3, we depict the dependence of $F_{C}^{D^{*+}}+F_{D}^{D^{*+}}$ on $M^{2}$ at two fixed values of $s_{0}$. Similar to the magnetic momentum case $F_{M}(0)$, the $F_{C}^{D^{*+}}+F_{D}^{D^{*+}}$ shows a weak dependency on the variation of $M^{2}$. Performing similar calculations for all vector and axial-vector mesons considered, we get the values of $F_{M}(0)$ and $F_{D}(0)$ presented in Tables III and IV, respectively.

The uncertainties result from the variation of Borel parameter $M^{2}$ and continuum threshold $s_{0}$ as well as from uncertainties in input parameters. All uncertainties

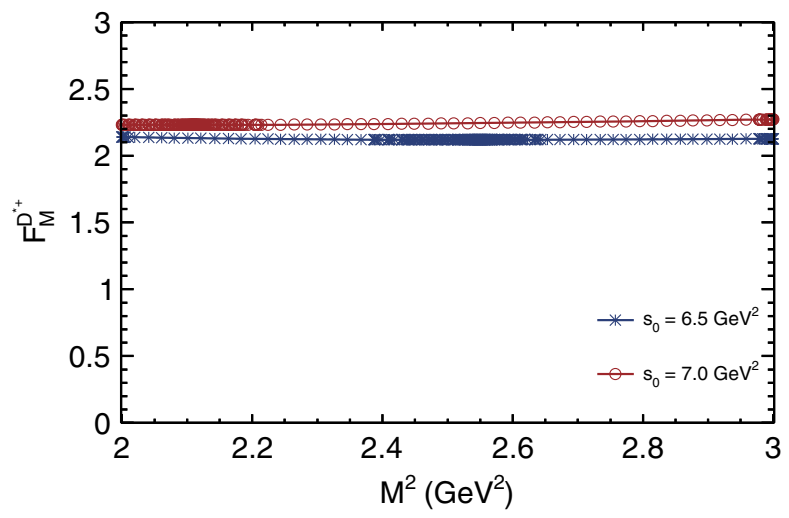

FIG. 1. The dependency of the magnetic moment of the $D^{*+}$ meson on $M^{2}$ at two fixed values of $s_{0}$.

are taken quadratically. Moreover, for completeness, in Table III, we also present the predictions on the magnetic moment obtained from the nonrelativistic (NR) quark model [54], Nambu-Jona-Lasinio (NJL) model [10], bag model [12], expanded bag model [58], and chiral perturbation theory (ChPT) [59].

We see that the values of the magnetic moments of $D^{*+}$ and $D_{s}^{*+}$ predicted by the light-cone sum rules framework are in good agreement with the other approaches. Once the

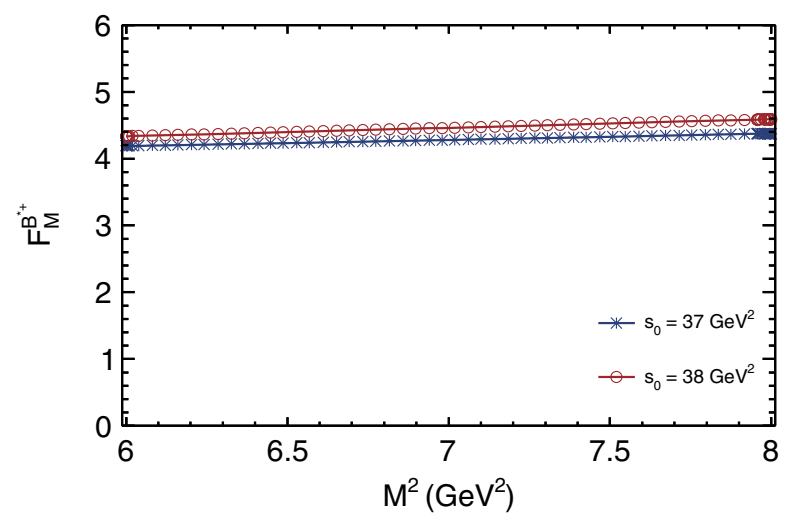

FIG. 2. The same as in Fig. 1, but for the $B^{*+}$ meson.

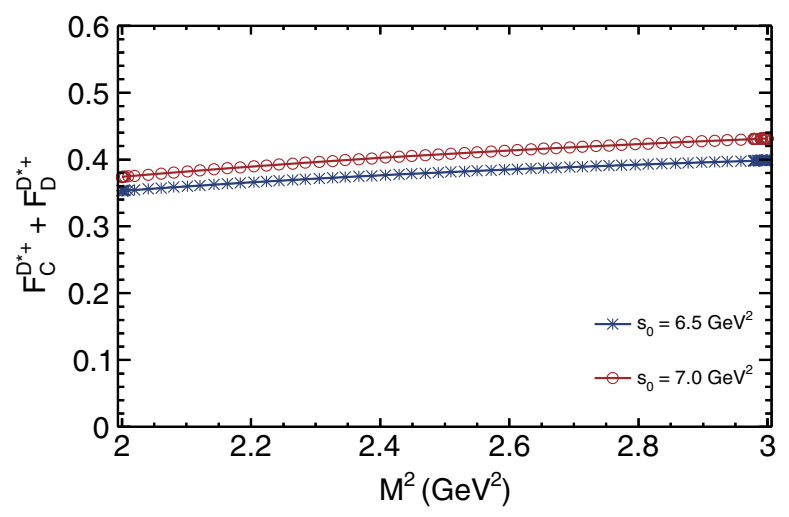

FIG. 3. The dependency of $F_{C}^{D^{*+}}+F_{D}^{D^{*+}}$ on $M^{2}$ for the $D^{*+}$ meson. 
TABLE III. Magnetic moments (in nuclear magneton) of heavy vector and axial-vector mesons.

\begin{tabular}{|c|c|c|c|c|c|c|}
\hline Particle & Our & NJL [10] & NR [56] & Bag [12] & Extended-bag [58] & ChPT [59] \\
\hline$\overline{D^{* 0}}$ & $(0.30 \pm 0.04)$ & $\cdots$ & -1.47 & -0.89 & -1.28 & $1.48_{-0.38}^{+0.22}$ \\
\hline$D^{*+}$ & $(1.16 \pm 0.08)$ & 1.16 & 1.32 & 1.17 & 1.13 & $1.62_{-0.08}^{+0.38}$ \\
\hline$D_{s}^{*}$ & $(1.00 \pm 0.14)$ & 0.98 & 1.00 & 1.03 & 0.93 & $0.69_{-010}^{+0.22}$ \\
\hline$B^{*+}$ & $(0.90 \pm 0.19)$ & 1.47 & 1.92 & 1.54 & 1.56 & $1.77_{-0.30}^{+0.25}$ \\
\hline$B^{* 0}$ & $-(0.21 \pm 0.04)$ & $\ldots$ & -0.87 & -0.64 & -0.69 & $-0.92_{-0.11}^{+0.30}$ \\
\hline$B_{s}^{*}$ & $-(0.17 \pm 0.02)$ & $\ldots$ & -0.55 & -0.47 & -0.51 & $-0.27_{-0.10}^{+0.13}$ \\
\hline$D_{1}^{0}$ & $(0.18 \pm 0.04)$ & $\cdots$ & $\ldots$ & $\ldots$ & $\ldots$ & $\ldots$ \\
\hline$D_{1}^{+}$ & $(0.90 \pm 0.08)$ & $\ldots$ & $\ldots$ & $\ldots$ & $\ldots$ & $\ldots$ \\
\hline$D_{s 1}^{1}$ & $(0.87 \pm 0.08)$ & $\ldots$ & $\ldots$ & $\ldots$ & $\ldots$ & $\ldots$ \\
\hline$B_{1}^{0}$ & $(0.14 \pm 0.08)$ & $\ldots$ & $\ldots$ & $\ldots$ & $\ldots$ & $\ldots$ \\
\hline$B_{1}^{+}$ & $(0.60 \pm 0.07)$ & $\ldots$ & $\ldots$ & $\ldots$ & $\ldots$ & $\ldots$ \\
\hline$B_{s 1}$ & $(0.13 \pm 0.09)$ & $\ldots$ & $\ldots$ & $\ldots$ & $\ldots$ & $\ldots$ \\
\hline
\end{tabular}

TABLE IV. The quadrupole moments of heavy vector and axial-vector mesons are depicted in natural units.

\begin{tabular}{lr}
\hline \hline & $F_{D}$ (in $e / m_{i}^{2}$ unit) \\
\hline$D^{* 0}$ & $(0.25 \pm 0.05)$ \\
$D^{*+}$ & $-(0.64 \pm 0.02)$ \\
$D_{s}^{*}$ & $-(0.60 \pm 0.02)$ \\
$B^{*+}$ & $-(0.80 \pm 0.10)$ \\
$B^{* 0}$ & $-(0.20 \pm 0.03)$ \\
$B_{s}^{* 0}$ & $-(0.17 \pm 0.03)$ \\
$D_{1}^{0}$ & $(0.18 \pm 0.02)$ \\
$D_{1}^{+}$ & $-(0.60 \pm 0.02)$ \\
$D_{s 1}$ & $-(0.59 \pm 0.02)$ \\
$B_{1}^{0}$ & $-(0.12 \pm 0.02)$ \\
$B_{1}^{+}$ & $-(0.78 \pm 0.02)$ \\
$B_{s 1}$ & $-(0.10 \pm 0.02)$ \\
\hline \hline
\end{tabular}

uncertainties are taken into account, our result on the $B_{s}^{* 0}$ magnetic moment is compatible with the prediction of the chiral perturbation theory.

$S U(3)$ symmetry dictates that the magnetic moments of $D^{*+}, D_{s}^{+}, B^{* 0}, B_{s}^{0}, D_{s 1}, D_{1}^{+}, B_{1}^{0}$, and $B_{s 1}^{0}$ should be very close to each other. Our predictions for the magnetic moments of these mesons are in good agreement within $S U(3)$ symmetry expectations. The violation of $S U(3)$ symmetry is about maximum $20 \%$. The violation of $S U(3)$ symmetry is due to the mass of the strange quark and the different values of quark condensates for $u, d$, and $s$ quarks as well as the values of the leptonic decay constants. However, predictions of the chiral perturbation theory [59] lead to a huge (4 times) violation of $S U(3)$ symmetry which seems highly unnatural.

The difference between our predictions and the other approaches on the magnetic moments can be explained as follows. The main contribution to the magnetic moments in light-cone sum rules results from the perturbative part of the spectral density. The perturbative part schematically can be written as

$$
\left(e_{Q}-e_{q}\right) A+e_{Q} B
$$

where numerically $A$ is larger than $B$. In the charged meson case, $e_{Q}-e_{q}$ is equal to one, and for this reason the magnetic moment is quite large. However, for the neutral meson case, $e_{Q}-e_{q}=0$, and, hence, the magnetic moment is rather small. Our last remark to this section is as follows. To increase the precision of our calculations, the next-toleading-order (NLO) QCD corrections to the correlation functions should be taken into account. In addition, the same order of NLO corrections should be included for the calculation of the leptonic decay constants. However, since the expressions for considered multipole moments depend on the ratio of these two factors, it is expected that our findings may not be changed considerably.

Finally, we emphasize that the magnetic moments of axial-vector heavy mesons are calculated for the first time to our knowledge. It would be interesting to have results within other approaches for the magnetic moments of these mesons.

\section{CONCLUSION}

The magnetic and quadrupole moments of vector and axial-vector mesons containing a heavy quark are estimated within the light-cone QCD sum rules framework by using photon distribution amplitudes. The magnetic moments of axial-vector mesons are estimated for the first time. Moreover, we compared our predictions on magnetic moments with the results obtained from other approaches. Our findings agree with the results of other methods for $D^{*+}$ and $D_{s}^{*}$ mesons. Besides, our predictions for the magnetic moments of $D^{*+}, D_{s}^{+}, B^{* 0}, B_{s}^{0}, D_{s 1}, D_{1}^{+}, B_{1}^{0}$, and $B_{s 1}^{0}$ mesons are consistent with $S U(3)$ symmetry expectations. However, the chiral perturbation theory predicts huge $S U(3)$ symmetry breaking (about 4 times), which is bizarre. The calculation of these magnetic moments for the axial-vector mesons within other approaches can be very useful for understanding the inner structure of heavy mesons. 


\section{APPENDIX A: PHOTON DISTRIBUTION AMPLITUDES}

In this Appendix, we present the matrix element of nonlocal operators in terms of photon DAs and their explicit expressions:

$$
\begin{aligned}
& \left\langle\gamma(q)\left|\bar{q}(x) \sigma_{\mu \nu} q(0)\right| 0\right\rangle=-i e_{q}\langle\bar{q} q\rangle\left(\varepsilon_{\mu} q_{\nu}-\varepsilon_{\nu} q_{\mu}\right) \int_{0}^{1} d u e^{i \bar{u} q x}\left(\chi \varphi_{\gamma}(u)+\frac{x^{2}}{16} \mathbb{A}(u)\right) \\
& -\frac{i}{2(q x)} e_{q}\langle\bar{q} q\rangle\left[x_{\nu}\left(\varepsilon_{\mu}-q_{\mu} \frac{\varepsilon x}{q x}\right)-x_{\mu}\left(\varepsilon_{\nu}-q_{\nu} \frac{\varepsilon x}{q x}\right)\right] \int_{0}^{1} d u e^{i \bar{u} q x} h_{\gamma}(u), \\
& \left\langle\gamma(q)\left|\bar{q}(x) \gamma_{\mu} q(0)\right| 0\right\rangle=e_{q} f_{3 \gamma}\left(\varepsilon_{\mu}-q_{\mu} \frac{\varepsilon x}{q x}\right) \int_{0}^{1} d u e^{i \bar{u} q x} \psi^{v}(u), \\
& \left\langle\gamma(q)\left|\bar{q}(x) \gamma_{\mu} \gamma_{5} q(0)\right| 0\right\rangle=-\frac{1}{4} e_{q} f_{3 \gamma} \epsilon_{\mu \nu \alpha \beta} \varepsilon^{\nu} q^{\alpha} x^{\beta} \int_{0}^{1} d u e^{i \bar{u} q x} \psi^{a}(u), \\
& \left\langle\gamma(q)\left|\bar{q}(x) g_{s} G_{\mu \nu}(v x) q(0)\right| 0\right\rangle=-i e_{q}\langle\bar{q} q\rangle\left(\varepsilon_{\mu} q_{\nu}-\varepsilon_{\nu} q_{\mu}\right) \int \mathcal{D} \alpha_{i} e^{i\left(\alpha_{\bar{q}}+v \alpha_{g}\right) q x} \mathcal{S}\left(\alpha_{i}\right), \\
& \left\langle\gamma(q)\left|\bar{q}(x) g_{s} \tilde{G}_{\mu \nu} i \gamma_{5}(v x) q(0)\right| 0\right\rangle=-i e_{q}\langle\bar{q} q\rangle\left(\varepsilon_{\mu} q_{\nu}-\varepsilon_{\nu} q_{\mu}\right) \int \mathcal{D} \alpha_{i} e^{i\left(\alpha_{\bar{q}}+v \alpha_{g}\right) q x} \tilde{\mathcal{S}}\left(\alpha_{i}\right), \\
& \left\langle\gamma(q)\left|\bar{q}(x) g_{s} \tilde{G}_{\mu \nu}(v x) \gamma_{\alpha} \gamma_{5} q(0)\right| 0\right\rangle=e_{q} f_{3 \gamma} q_{\alpha}\left(\varepsilon_{\mu} q_{\nu}-\varepsilon_{\nu} q_{\mu}\right) \int \mathcal{D} \alpha_{i} e^{i\left(\alpha_{\bar{q}}+v \alpha_{g}\right) q x} \mathcal{A}\left(\alpha_{i}\right), \\
& \left\langle\gamma(q)\left|\bar{q}(x) g_{s} G_{\mu \nu}(v x) i \gamma_{\alpha} q(0)\right| 0\right\rangle=e_{q} f_{3 \gamma} q_{\alpha}\left(\varepsilon_{\mu} q_{\nu}-\varepsilon_{\nu} q_{\mu}\right) \int \mathcal{D} \alpha_{i} e^{i\left(\alpha_{\bar{q}}+v \alpha_{g}\right) q x} \mathcal{V}\left(\alpha_{i}\right), \\
& \left\langle\gamma(q)\left|\bar{q}(x) \sigma_{\alpha \beta} g_{s} G_{\mu \nu}(v x) q(0)\right| 0\right\rangle \\
& =e_{q}\langle\bar{q} q\rangle\left\{\left[\left(\varepsilon_{\mu}-q_{\mu} \frac{\varepsilon x}{q x}\right)\left(g_{\alpha \nu}-\frac{1}{q x}\left(q_{\alpha} x_{\nu}+q_{\nu} x_{\alpha}\right)\right) q_{\beta}-\left(\varepsilon_{\mu}-q_{\mu} \frac{\varepsilon x}{q x}\right)\left(g_{\beta \nu}-\frac{1}{q x}\left(q_{\beta} x_{\nu}+q_{\nu} x_{\beta}\right)\right) q_{\alpha}\right.\right. \\
& \left.-\left(\varepsilon_{\nu}-q_{\nu} \frac{\varepsilon x}{q x}\right)\left(g_{\alpha \mu}-\frac{1}{q x}\left(q_{\alpha} x_{\mu}+q_{\mu} x_{\alpha}\right)\right) q_{\beta}+\left(\varepsilon_{\nu}-q_{\nu} \frac{\varepsilon x}{q x}\right)\left(g_{\beta \mu}-\frac{1}{q x}\left(q_{\beta} x_{\mu}+q_{\mu} x_{\beta}\right)\right) q_{\alpha}\right] \int \mathcal{D} \alpha_{i} e^{i\left(\alpha_{\bar{q}}+v \alpha_{g}\right) q x} \mathcal{T}_{1}\left(\alpha_{i}\right) \\
& +\left[\left(\varepsilon_{\alpha}-q_{\alpha} \frac{\varepsilon x}{q x}\right)\left(g_{\mu \beta}-\frac{1}{q x}\left(q_{\mu} x_{\beta}+q_{\beta} x_{\mu}\right)\right) q_{\nu}-\left(\varepsilon_{\alpha}-q_{\alpha} \frac{\varepsilon x}{q x}\right)\left(g_{\nu \beta}-\frac{1}{q x}\left(q_{\nu} x_{\beta}+q_{\beta} x_{\nu}\right)\right) q_{\mu}\right. \\
& \left.-\left(\varepsilon_{\beta}-q_{\beta} \frac{\varepsilon x}{q x}\right)\left(g_{\mu \alpha}-\frac{1}{q x}\left(q_{\mu} x_{\alpha}+q_{\alpha} x_{\mu}\right)\right) q_{\nu}+\left(\varepsilon_{\beta}-q_{\beta} \frac{\varepsilon x}{q x}\right)\left(g_{\nu \alpha}-\frac{1}{q x}\left(q_{\nu} x_{\alpha}+q_{\alpha} x_{\nu}\right)\right) q_{\mu}\right] \int \mathcal{D} \alpha_{i} e^{i\left(\alpha_{\bar{q}}+v \alpha_{g}\right) q x} \mathcal{T}_{2}\left(\alpha_{i}\right) \\
& \left.+\frac{1}{q x}\left(q_{\mu} x_{\nu}-q_{\nu} x_{\mu}\right)\left(\varepsilon_{\alpha} q_{\beta}-\varepsilon_{\beta} q_{\alpha}\right) \int \mathcal{D} \alpha_{i} e^{i\left(\alpha_{\bar{q}}+v \alpha_{g}\right) q x} \mathcal{T}_{3}\left(\alpha_{i}\right)+\frac{1}{q x}\left(q_{\alpha} x_{\beta}-q_{\beta} x_{\alpha}\right)\left(\varepsilon_{\mu} q_{\nu}-\varepsilon_{\nu} q_{\mu}\right) \int \mathcal{D} \alpha_{i} e^{i\left(\alpha_{\bar{q}}+v \alpha_{g}\right) q x} \mathcal{T}_{4}\left(\alpha_{i}\right)\right\},
\end{aligned}
$$

where $\chi$ is the magnetic susceptibility of the quarks, $\varphi_{\gamma}(u)$ is the leading twist $2, \psi^{v}(u), \psi^{a}(u), \mathcal{A}$, and $\mathcal{V}$ are the twist 3 , and $h_{\gamma}(u), \mathbb{A}$, and $\mathcal{T}_{i}(i=1,2,3,4)$ are the twist 4 photon distribution amplitudes. The integral measure $\mathcal{D} \alpha_{i}$ is defined as

$$
\mathcal{D} \alpha_{i}=\int_{0}^{1} d \alpha_{g} \int_{0}^{1} d \alpha_{q} \int_{0}^{1} d \alpha_{\bar{q}} \delta\left(1-\alpha_{g}-\alpha_{q}-\alpha_{\bar{q}}\right)
$$

The expressions of the photon DAs which we need in our calculations are [49]

$$
\begin{aligned}
\varphi_{\gamma}(u) & =6 u \bar{u}\left[1+\varphi_{2}(\mu) C_{2}^{3 / 2}(u-\bar{u})\right], \\
\psi^{v}(u) & =3\left[3(2 u-1)^{2}-1\right]+\frac{3}{64}\left(15 w_{\gamma}^{V}-5 w_{\gamma}^{A}\right)\left[3-30(2 u-1)^{2}+35(2 u-1)^{4}\right], \\
\psi^{a}(u) & =\left[1-(2 u-1)^{2}\right]\left[5(2 u-1)^{2}-1\right] \frac{5}{2}\left(1+\frac{9}{16} w_{\gamma}^{V}-\frac{3}{16} w_{\gamma}^{A}\right),
\end{aligned}
$$


TABLE V. The values of the constant parameters entering into the distribution amplitudes at the renormalization scale $\mu=1 \mathrm{GeV}$.

\begin{tabular}{cccccccccc}
\hline \hline$\varphi_{2}$ & $\kappa$ & $\kappa^{+}$ & $\xi_{1}$ & $\xi_{1}^{+}$ & $\xi_{2}$ & $\xi_{2}^{+}$ & $f_{3 \gamma}\left(\mathrm{GeV}^{2}\right)$ & $\omega_{\gamma}^{V}$ & $\omega_{\gamma}^{A}$ \\
\hline 0.0 & 0.2 & 0.0 & 0.4 & 0.0 & 0.3 & 0.0 & $(-4.0 \pm 2.0) \times 10^{-3}$ & $(3.8 \pm 1.8)$ & $(-2.1 \pm 1.0)$ \\
\hline \hline
\end{tabular}

$$
\begin{aligned}
\mathcal{A}\left(\alpha_{i}\right)= & 360 \alpha_{q} \alpha_{\bar{q}} \alpha_{g}^{2}\left[1+w_{\gamma}^{A} \frac{1}{2}\left(7 \alpha_{g}-3\right)\right], \\
\mathcal{V}\left(\alpha_{i}\right)= & 540 w_{\gamma}^{V}\left(\alpha_{q}-\alpha_{\bar{q}}\right) \alpha_{q} \alpha_{\bar{q}} \alpha_{g}^{2}, \\
h_{\gamma}(u)= & -10\left(1+2 \kappa^{+}\right) C_{2}^{1 / 2}(u-\bar{u}), \\
\mathbb{A}(u)= & 40 u^{2} \bar{u}^{2}\left(3 \kappa-\kappa^{+}+1\right)+8\left(\zeta_{2}^{+}-3 \zeta_{2}\right)\left[u \bar{u}(2+13 u \bar{u})+2 u^{3}\left(10-15 u+6 u^{2}\right) \ln (u)\right. \\
& \left.+2 \bar{u}^{3}\left(10-15 \bar{u}+6 \bar{u}^{2}\right) \ln (\bar{u})\right], \\
\mathcal{T}_{1}\left(\alpha_{i}\right)= & -120\left(3 \zeta_{2}+\zeta_{2}^{+}\right)\left(\alpha_{\bar{q}}-\alpha_{q}\right) \alpha_{\bar{q}} \alpha_{q} \alpha_{g}, \\
\mathcal{T}_{2}\left(\alpha_{i}\right)= & 30 \alpha_{g}^{2}\left(\alpha_{\bar{q}}-\alpha_{q}\right)\left[\left(\kappa-\kappa^{+}\right)+\left(\zeta_{1}-\zeta_{1}^{+}\right)\left(1-2 \alpha_{g}\right)+\zeta_{2}\left(3-4 \alpha_{g}\right)\right], \\
\mathcal{T}_{3}\left(\alpha_{i}\right)= & -120\left(3 \zeta_{2}-\zeta_{2}^{+}\right)\left(\alpha_{\bar{q}}-\alpha_{q}\right) \alpha_{\bar{q}} \alpha_{q} \alpha_{g}, \\
\mathcal{T}_{4}\left(\alpha_{i}\right)= & 30 \alpha_{g}^{2}\left(\alpha_{\bar{q}}-\alpha_{q}\right)\left[\left(\kappa+\kappa^{+}\right)+\left(\zeta_{1}+\zeta_{1}^{+}\right)\left(1-2 \alpha_{g}\right)+\zeta_{2}\left(3-4 \alpha_{g}\right)\right], \\
\mathcal{S}\left(\alpha_{i}\right)= & 30 \alpha_{g}^{2}\left\{\left(\kappa+\kappa^{+}\right)\left(1-\alpha_{g}\right)+\left(\zeta_{1}+\zeta_{1}^{+}\right)\left(1-\alpha_{g}\right)\left(1-2 \alpha_{g}\right)+\zeta_{2}\left[3\left(\alpha_{\bar{q}}-\alpha_{q}\right)^{2}-\alpha_{g}\left(1-\alpha_{g}\right)\right]\right\}, \\
\tilde{\mathcal{S}}\left(\alpha_{i}\right)= & -30 \alpha_{g}^{2}\left\{\left(\kappa-\kappa^{+}\right)\left(1-\alpha_{g}\right)+\left(\zeta_{1}-\zeta_{1}^{+}\right)\left(1-\alpha_{g}\right)\left(1-2 \alpha_{g}\right)+\zeta_{2}\left[3\left(\alpha_{\bar{q}}-\alpha_{q}\right)^{2}-\alpha_{g}\left(1-\alpha_{g}\right)\right]\right\},
\end{aligned}
$$

where $C_{n}^{m}$ is the Gegenbauer polynomial. The constants entering the above DAs are adapted from Ref. [49], and their values are given in Table V.

\section{APPENDIX B: EXPLICIT EXPRESSIONS OF THE INVARIANT FUNCTIONS}

In this Appendix, we present the explicit expressions of the invariant functions $\Pi_{1}^{( \pm)}, \Pi_{2}^{( \pm)}$, and $\Pi_{3}^{( \pm)}$.

\section{Coefficient of the $(\varepsilon \cdot p) p_{\mu} p_{\nu}$ structure}

$$
\begin{aligned}
\Pi_{1}^{( \pm)}= & \left.-\frac{1}{\pi^{2}}\left[3\left(e_{Q}-e_{q}\right) m_{Q}^{4} M^{2}\left(\mathcal{I}_{3}-m_{Q}^{2} \mathcal{I}_{4}\right)\right]-\frac{e^{-m_{b}^{2} / M^{2}}}{24 \pi^{2} M^{2}}\left[48 \pi^{2} e_{Q} m_{q}\langle\bar{q} q\rangle+e^{m_{b}^{2} / M^{2}} e_{q}\left\langle g_{s}^{2} G^{2}\right\rangle m_{Q}^{2} \mathcal{I}_{2}\right)\right] \\
& +\frac{e^{-m_{b}^{2} / M^{2}}}{3 M^{4}} e_{Q} m_{0}^{2} m_{q}\langle\bar{q} q\rangle+\frac{e^{-m_{b}^{2} / M^{2}}}{3 M^{6}} e_{Q} m_{0}^{2} m_{Q}^{2} m_{q}\langle\bar{q} q\rangle
\end{aligned}
$$

\section{Coefficient of the $(\varepsilon \cdot p) p_{\nu} q_{\mu}$ structure}

$$
\begin{aligned}
\Pi_{2}^{( \pm)}= & -\frac{3}{4 \pi^{2}} m_{Q}^{2} M^{2}\left[e_{Q} \mathcal{I}_{2}+\left(e_{Q}-3 e_{q}\right) m_{Q}^{2} \mathcal{I}_{3}-2\left(e_{Q}-e_{q}\right) m_{Q}^{4} \mathcal{I}_{4}\right] \\
& -\frac{e^{-m_{b}^{2} / M^{2}}}{96 \pi^{2} M^{2}}\left[e_{q}\left\langle g_{s}^{2} G^{2}\right\rangle+144 e_{Q} m_{q} \pi^{2}\langle\bar{q} q\rangle+2 e^{m_{b}^{2} / M^{2}} e_{q}\left\langle g_{s}^{2} G^{2}\right\rangle m_{Q}^{2} \mathcal{I}_{2}\right] \pm \frac{e^{-m_{b}^{2} / M^{2}}}{M^{2}}\left[2 e_{q} m_{Q}\langle\bar{q} q\rangle \tilde{j}_{2}\left(h_{\gamma}\right)\right] \\
& +\frac{e^{-m_{b}^{2} / M^{2}}}{6 M^{4}} e_{Q} m_{0}^{2} m_{q}\langle\bar{q} q\rangle+\frac{e^{-m_{b}^{2} / M^{2}}}{144 M^{6}} m_{Q}\left\{36 e_{Q} m_{0}^{2} m_{Q} m_{q}\langle\bar{q} q\rangle\right. \\
& \left.+e_{q}\left\langle g_{s}^{2} G^{2}\right\rangle\left[ \pm 12\langle\bar{q} q\rangle \tilde{j}_{2}\left(h_{\gamma}\right)+f_{3 \gamma} m_{Q}\left(4 \tilde{j}_{1}\left(\psi^{v}\right)+\psi^{a}\left(u_{0}\right)\right)\right]\right\} \\
& \mp \frac{e^{-m_{b}^{2} / M^{2}}}{36 M^{8}} e_{q}\left\langle g_{s}^{2} G^{2}\right\rangle m_{Q}^{3}\langle\bar{q} q\rangle \tilde{j}_{2}\left(h_{\gamma}\right)-\frac{e^{-m_{b}^{2} / M^{2}}}{2} e_{q} f_{3 \gamma}\left[4 \tilde{j}_{1}\left(\psi^{v}\right)+\psi^{a}\left(u_{0}\right)\right] .
\end{aligned}
$$




\section{Coefficient of the $(\varepsilon \cdot p) q_{\mu} q_{\nu}$ structure}

$$
\begin{aligned}
\Pi_{3}^{( \pm)}= & -\frac{1}{4 \pi^{2}}\left[3\left(e_{Q}-e_{q}\right) m_{Q}^{4} M^{2}\left(\mathcal{I}_{3}-m_{Q}^{2} \mathcal{I}_{4}\right)\right]-\frac{e^{-m_{b}^{2} / M^{2}}}{96 M^{2} \pi^{2}}\left\{48 e_{Q} m_{q} \pi^{2}\langle\bar{q} q\rangle+e^{m_{b}^{2} / M^{2}} e_{q}\left\langle g_{s}^{2} G^{2}\right\rangle m_{Q}^{2} \mathcal{I}_{2}\right. \\
& \left. \pm 384 e_{q} m_{Q} \pi^{2}\langle\bar{q} q\rangle\left[i_{1}^{\prime}\left(\mathcal{T}_{1}, 1\right)+i_{1}^{\prime}\left(\mathcal{T}_{2}, 1\right)-i_{1}^{\prime}\left(\mathcal{T}_{3}, 1\right)-i_{1}^{\prime}\left(\mathcal{T}_{4}, 1\right)\right]\right\} \\
& +\frac{e^{-m_{b}^{2} / M^{2}}}{12 M^{4}} e_{Q} m_{0}^{2} m_{q}\langle\bar{q} q\rangle+\frac{e^{-m_{b}^{2} / M^{2}}}{36 M^{6}}\left\{m_{Q}^{2}\left[3 e_{Q} m_{0}^{2} m_{q}\langle\bar{q} q\rangle+e_{q} f_{3 \gamma}\left\langle g_{s}^{2} G^{2}\right\rangle \tilde{j}_{1}\left(\psi^{v}\right)\right]\right\} \\
& +e^{-m_{b}^{2} / M^{2}}\left\{2 e_{q} f_{3 \gamma}\left[2 i_{2}(\mathcal{V}, v)+i_{2}^{\prime}(\mathcal{A}, 1)-i_{2}^{\prime}(\mathcal{V}, 1)-\tilde{j}_{1}\left(\psi^{v}\right)\right]\right\},
\end{aligned}
$$

where

$$
\begin{aligned}
\mathcal{I}_{n} & =\int_{m_{b}^{2}}^{s_{0}} d s \frac{e^{-s / M^{2}}}{s^{n}}, \\
i_{1}(\phi, f(v)) & =\int \mathcal{D} \alpha_{i} \int_{0}^{1} d v \phi\left(\alpha_{\bar{q}}, \alpha_{q}, \alpha_{g}\right) f(v) \theta\left(k-u_{0}\right), \\
i_{2}(\phi, f(v)) & =\int \mathcal{D} \alpha_{i} \int_{0}^{1} d v \phi\left(\alpha_{\bar{q}}, \alpha_{q}, \alpha_{g}\right) f(v) \delta\left(k-u_{0}\right), \\
i_{1}^{\prime}(\phi, f(v)) & =\int \mathcal{D} \alpha_{i} \int_{0}^{1} d v \phi\left(\alpha_{\bar{q}}, \alpha_{q}, \alpha_{g}\right) f(v) \theta\left(k^{\prime}-u_{0}\right), \\
i_{2}^{\prime}(\phi, f(v)) & =\int \mathcal{D} \alpha_{i} \int_{0}^{1} d v \phi\left(\alpha_{\bar{q}}, \alpha_{q}, \alpha_{g}\right) f(v) \delta\left(k^{\prime}-u_{0}\right), \\
\tilde{j}_{1}(f(u)) & =\int_{u_{0}}^{1} d u f(u), \\
\tilde{j}_{2}(f(u)) & =\int_{u_{0}}^{1} d u\left(u-u_{0}\right) f(u),
\end{aligned}
$$

and

$$
k=\alpha_{q}+\alpha_{g} v, \quad k^{\prime}=\alpha_{q}+\alpha_{g}(1-v), \quad M^{2}=\frac{M_{1}^{2} M_{2}^{2}}{M_{1}^{2}+M_{2}^{2}}, \quad u_{0}=\frac{M_{1}^{2}}{M_{1}^{2}+M_{2}^{2}} .
$$

In calculations, we take $M_{1}^{2}=M_{2}^{2}$, since the initial and final state mesons are the same; hence, $u_{0}=\frac{1}{2}$.

[1] T. M. Aliev, A. Özpineci, and M. Savcı, Phys. Lett. B 678, 470 (2009).

[2] F. X. Lee, S. Moerschbacher, and W. Wilcox, Phys. Rev. D 78, 094502 (2008).

[3] J. N. Hedditch, W. Kamleh, B. G. Lasscock, D. B. Leinweber, A. G. Williams, and J. M. Zanotti, Phys. Rev. D 75, 094504 (2007).

[4] A. Samsonov, J. High Energy Phys. 02 (2003) 061.

[5] A. M. Badalian and Y. A. Simonov, Phys. Rev. D 87, 074012 (2013).

[6] M. S. Bhagwat and P. Maris, Phys. Rev. C 77, 025203 (2008).

[7] B. L. Bakker, H.-M. Choi, and C.-R. Ji, Phys. Rev. D 65, 116001 (2002).
[8] H.-M. Choi and C.-R. Ji, Phys. Rev. D 70, 053015 (2004).

[9] F. T. Hawes and M. A. Pichowsky, Phys. Rev. C 59, 1743 (1999).

[10] Y.-L. Luan, X.-L. Chen, and W.-Z. Deng, Chin. Phys. C 39, 113103 (2015).

[11] D. Djukanovic, E. Epelbaum, J. Gegelia, and U. G. Meißner, Phys. Lett. B 730, 115 (2014).

[12] S. K. Bose and L. P. Singh, Phys. Rev. D 22, 773 (1980).

[13] T. A. Lähde, Nucl. Phys. A714, 183 (2003).

[14] G. Toledo Sánchez and D. García Gudiño, Int. J. Mod. Phys. Conf. Ser. 35, 1460463 (2014).

[15] P. Colangelo, F. De Fazio, and G. Nardulli, Phys. Lett. B 316, 555 (1993).

[16] W. Jaus, Phys. Rev. D 53, 1349 (1996). 
[17] N. Brambilla, Y. Jia, and A. Vairo, Phys. Rev. D 73, 054005 (2006).

[18] S. S. Gershtein, V. V. Kiselev, A. K. Likhoded, and A. V. Tkabladze, Phys. Rev. D 51, 3613 (1995).

[19] L. P. Fulcher, Phys. Rev. D 60, 074006 (1999).

[20] T. Barnes, S. Godfrey, and E. S. Swanson, Phys. Rev. D 72, 054026 (2005).

[21] O. Lakhina and E.S. Swanson, Phys. Lett. B 650, 159 (2007).

[22] W. Wilcox, O. V. Maxwell, and K. A. Milton, Phys. Rev. D 31, 1081 (1985).

[23] Y.S. Zhong, T. S. Cheng, and A. W. Thomas, Nucl. Phys. A559, 579 (1993).

[24] A. Hiorth Örsland and H. Högaasen, Eur. Phys. J. C 9, 503 (1999).

[25] P. J. O'Donnell and Q. P. Xu, Phys. Lett. B 336, 113 (1994).

[26] F. Cardarelli, I. L. Grach, I. M. Narodetskii, G. Salmé, and S. Simula, Phys. Lett. B 359, 1 (1995).

[27] H.-M. Choi, Phys. Rev. D 75, 073016 (2007).

[28] M. Koll, R. Ricken, D. Merten, B. C. Metsch, and H. R. Petry, Eur. Phys. J. A 9, 73 (2000).

[29] T. A. Lähde, C. J. Nyfält, and D. O. Riska, Nucl. Phys. A674, 141 (2000).

[30] A. Y. Khodjamirian, Phys. Lett. B 90, 460 (1980).

[31] H. G. Dosch and S. Narison, Phys. Lett. B 368, 163 (1996).

[32] T. M. Aliev, E. Iltan, and N. K. Pak, Phys. Lett. B 334, 169 (1994).

[33] T. M. Aliev, D. A. Demir, E. Iltan, and N. K. Pak, Phys. Rev. D 54, 857 (1996).

[34] M. A. Shifman, Z. Phys. C 4, 345 (1980).

[35] T. M. Aliyev, Z. Phys. C 26, 275 (1984).

[36] V. A. Beilin and A. V. Radyushkin, Nucl. Phys. B260, 61 (1985).

[37] T. M. Aliev, E. Iltan, and N. K. Pak, Phys. Lett. B 329, 123 (1994).

[38] P. Colangelo, F. De Fazio, and A. Ozpineci, Phys. Rev. D 72, 074004 (2005).

[39] D. Bećirević and B. Haas, Eur. Phys. J. C 71, 1734 (2011).
[40] G. C. Donald, C. T. H. Davies, R. J. Dowdall, E. Follana, K. Hornbostel, J. Koponen, G. P. Lepage, and C. McNeile, Phys. Rev. D 86, 094501 (2012).

[41] P. Cho and H. Georgi, Phys. Lett. B 296, 408 (1992).

[42] J. F. Amundson, C. G. Boyd, E. Jenkins, M. Luke, A. V. Manohar, J. L. Rosner, M. J. Savage, and M. B. Wise, Phys. Lett. B 296, 415 (1992).

[43] W. A. Bardeen, E. J. Eichten, and C. T. Hill, Phys. Rev. D 68, 054024 (2003).

[44] A. Hiorth and J. O. Eeg, Eur. Phys. J. C 39, 27 (2005).

[45] Y.-H. Chen, Z.-H. Guo, and B.-S. Zou, Phys. Rev. D 91, 014010 (2015).

[46] V. M. Braun, in Proceedings of the 4th International Workshop on Progress in Heavy Quark Physics, Rostock, Germany (1997), pp. 105-118, https://labs.inspirehep.net/ literature/452985.

[47] S. J. Brodsky and J. R. Hiller, Phys. Rev. D 46, 2141 (1992).

[48] J. Rohrwild, J. High Energy Phys. 09 (2007) 073.

[49] P. Ball, V. M. Braun, and N. Kivel, Nucl. Phys. B649, 263 (2003).

[50] I. Balitsky and V. Braun, Nucl. Phys. B311, 541 (1989).

[51] V. M. Belyaev, V. M. Braun, A. Khodjamirian, and R. Rückl, Phys. Rev. D 51, 6177 (1995).

[52] K. G. Chetyrkin, A. Khodjamirian, and A. A. Pivovarov, Phys. Lett. B 661, 250 (2008).

[53] V. M. Braun and I. E. Filyanov, Z. Phys. C 48, 239 (1990); Yad. Fiz. 52, 199 (1990) [Sov. J. Nucl. Phys. 52, 126 (1990)].

[54] A. Khodjamirian, C. Klein, T. Mannel, and N. Offen, Phys. Rev. D 80, 114005 (2009).

[55] V. M. Belyaev and B. L. Ioffe, Zh. Eksp. Teor. Fiz. 83, 876 (1982) [Sov. Phys. JETP 56, 493 (1982)].

[56] M. Tanabashi et al. (Particle Data Group), Phys. Rev. D 98 , 030001 (2018).

[57] Z.-G. Wang, Eur. Phys. J. C 75, 427 (2015).

[58] V. Šimonis, Eur. Phys. J. A 52, 90 (2016).

[59] B. Wang, B. Yang, L. Meng, and S.-L. Zhu, Phys. Rev. D 100, 016019 (2019). 